\author{
A.S. Makhmet ${ }^{1}$, M.G. Sharaev ${ }^{2}$, \\ A.E. Dyusembaev ${ }^{1}$, A.M. Kustubayeva ${ }^{1 *}$ \\ ${ }^{1}$ Al-Farabi Kazakh National University, Almaty, Kazakhstan \\ ${ }^{2}$ Skolkovo Institute of Science and Technology, Moscow, Russia \\ *e-mail: almkusto@kaznu.kz
}

\title{
Machine learning for brain signal analysis
}

\begin{abstract}
Machine learning (ML) is an effective tool for analysing signals from the human brain. Machine Learning techniques provide new insight into the understanding of brain function in healthy subjects and patients with neurological and mental disorders. Here we introduce the application of machine learning to brain signal analysis, specifically using two widely used brain signal collection methods: functional magnetic resonance imaging (fMRI) and Electroencephalography (EEG). The article provides a brief overview of the theoretical concept of machine learning and its types: supervised, unsupervised and reinforcement learning. The potential of machine learning applications in pathology is discussed. Differences between EEG and fMRI methods regarding machine learning application and an overview of the techniques employed in different research studies are reviewed. The new machine learning methods invented for analysis of brain signals in the resting state and during the performance of the different cognitive tasks would be useful and worth considering in other domains, not limited to medicine.
\end{abstract}

Key words: EEG, fMRI, machine learning, MVPA, brain signal analysis.

\section{Introduction}

Machine learning applications are effective in solving many modern problems. Its widespread use has induced the emergence of a large amount of literature. The present article aims to provide a brief introduction to machine learning techniques and its application in brain signals analysis, specifically to fMRI and EEG signals.

First, we provide a brief overview of the theoretical concept of machine learning. Machine learning is a scientific discipline in which techniques are designed so that machines can computationally efficiently extract patterns, structures or relationships from data. It is a relatively new discipline, which lies at the intersection of two fields: mathematics and computer science. The majority of machine learning techniques are based on the following mathematical realms: linear algebra, analytic geometry, matrix decomposition, probability theory, vector calculus and optimization [1].

A learning process outcome would be called a model. Machine learning models describe relationships within observed data. A typical dataset that is considered in ML problems consists of dependent and independent variables. Independent variables can also be referred to as features, while a dependent variable, which depends on independent variables, is referred to as a target.

Machine learning techniques are based on regression, dimensionality reduction, density estimation and classification [1]. The key objective in regression and classification problems is to build a model that would map inputs $x$ to corresponding values $y$, where $x$ represents feature matrix and $y$ represents a target vector [1]. The difference is that in regression the outputs are continuous values, whereas in classification the outputs are discrete (or categorical) values. The key objective in dimensionality reduction problems is to reduce the number of features in the feature matrix, with minimum loss of potentially valuable information [1]. The key objective in density estimation problems is to describe a dataset from the perspective of a probability distribution [1].

Nowadays there are many different types of learning that exist in Machine learning. Traditionally, machine learning approaches are divided into three broad categories: supervised, unsupervised, and reinforcement learning. Reinforcement learning is a type of learning, in which an 'agent' learns how to act in an environment by continuously getting 'feedback' from that environment. In supervised learning, a model is trained on labeled data to learn a relationship between features and a target variable. Next, to 
evaluate the performance of the model, the model is tested on previously 'unseen' data. Model training is the process during which a machine learns patterns and structure from available data. In contrast to the supervised learning approach, the unsupervised approach works with unlabeled data, and a model is built to discover patterns and structures within the data. Supervised learning is used in regression and classification algorithms, whereas unsupervised learning is used in dimensionality reduction, density estimation, anomaly detection, autoencoding and clustering techniques. In supervised learning, we are able to evaluate our model by comparing predicted and actual labels of test data. In contrast, in unsupervised learning there are no labels to predict, and, therefore, there is no direct evaluation for unsupervised learning. However, an output of an unsupervised learning task is often used to construct an input (informative features) to the subsequent supervised learning task, and it can be evaluated via results of the subsequent supervised learning task and answer the question, "Was the pattern discovered in unsupervised learning useful?" [2].

\section{Machine learning application in clinics}

Medicine and pathology in particular are argWuably the most promising domains to apply machine learning: what could be more inspiring than contributing to saving millions of lives from complex diseases or improving the quality of life of those who are paralyzed? With each year there is an increased number of publications presenting applications of machine learning algorithms to medical data, yet this has not resulted in many meaningful contributions to clinical care [2]. The main reason is possibly low $\mathrm{AI} / \mathrm{ML}$ algorithms trustworthiness, which comprises several parts: is the algorithm accurate and robust, how fair and transparent are its decisions, and finally, how interpretable are these decisions to the medical community [3]. While high accuracy and robustness (for example to low quality data) are well-known desired properties of AI/ML systems in all applied fields, model interpretability becomes crucial, especially in a clinical setting. Another factor that makes it difficult to apply machine learning to medical data is that acquisition of data is a costly and lengthy process. For example, for brain signal analysis to acquire data using fMRI, one must have an fMRI scanner, which is considered to be expensive, and not many research groups can afford it. Comparatively, getting other types of data is much easier. For example, Netflix possesses all the data that is needed in order

Int. j. biol. chem. (Online) to build its own recommendation system, because it analyses traffic that a user generates when interacting with a Netflix website or application.

Both supervised and unsupervised learning are used in pathology and human functions. One example is analysis of data obtained from patients with Heart Failure with preserved Ejection Fraction (HFpEF) [2]. HFpEF is a complex condition and reflects multiple dominant pathophysiologic processes. The idea of the analysis was to group patients on the basis of qualitative echocardiographic and clinical variables. Initially, there were 67 different features; after removing highly correlated features, there were 46 predictors (features). Next, a regularized form of a clustering algorithm was applied: clusters were determined by using multivariate Gaussian distributions and using means and standard deviation assigned to each feature [2]. The clusters were formed by calculating a joint probability of membership for each patient. Results of the comparison of the calculated clusters have demonstrated the differences across many phenotypic variables. These phenotypic clusters resulted in becoming features in the supervised learning model that predicted survival of HFpEF patients.

Study of signals of the brain, the most complex structure in the body, may help understand brain functioning in normal versus neurological or mental disorder brain conditions. Many studies use supervised learning approaches to diagnose a brain function pathology or classify symptom severity from concurrent neuroimaging data. Among brain function disorders are attention-deficit/hyperactivity disorder, autism, depression, and schizophrenia [4]. Moreover, machine learning technology is able to detect deviations from normative development trajectories as risk factors for psychopathology. Defining an age of an individual based on brain activity network patterns can be used to elucidate atypical development in children and adults with Tourette syndrome [4]. There is a growing number of studies using brain connectivity approaches [5]. This method is based on graph theory and determines functional, structural, and causal dynamical networks. Therefore, brain connectivity measurements appear to serve as variables to determine whether it is possible to predict subsequent diagnosis or treatment outcomes.

Apart from supervised learning in brain signal analysis, an unsupervised learning approach can be used to cluster patients into subgroups with categorically different patterns of neuroimaging features. The application of reinforcement learning is also considered in the field of neuroscience [6-7]. It is worth mentioning that the Reinforcement learning 
approach itself gets its aspiration from the cognitive neuroscience field, as it tries to mimic brain function.

There are different techniques for measuring and mapping brain activity. These include Electroencephalography (EEG), functional magnetic resonance imaging (fMRI), Positron emission tomography (PET) and Magnetoencephalography (MEG). Our focus in this article is on the two most popular noninvasive and safe methods to obtain brain signals during the cognitive task or resting states: fMRI and EEG.

\section{Machine learning in functional MRI signal analysis}

FMRI measures brain neural activity via magnetic properties of blood, namely, the blood-oxygen level dependent (BOLD) signal. The method is based on the fact that with an increase in the activity of a particular area of the brain, blood flow to this area also increases, which means that the parameters of blood movement and level of oxygen in the vascular bed change. A typical neuroimaging experiment holds for several sessions (runs) per subject. In fMRI, the whole brain is scanned for the duration of a session, resulting in many brain images per time unit, called volumes. The scanning rate affects the spatial and temporal resolutions of images. FMRI has good spatial resolution and a satisfactory level of temporal resolution, even though these spatial and temporal resolutions are attained at the expense of each other.

For one to perform an effective fMRI analysis, the data collected should first undergo the preprocessing stage. Preprocessing typically includes Realigning and Unwarping the Data, Slice-time correction, Co-registration, Segmentation, Normalization, and Smoothing steps. The last Smoothing step is in many cases omitted to avoid distortion of neural activity intensity per voxel.

The first types of analyses that prevailed in the analysis of fMRI data were univariate and massunivariate. In univariate analysis, an amplitude of a signal elicited from a voxel, which is a 3-dimensional pixel of a brain (usually $3 \mathrm{~mm} * 3 \mathrm{~mm} * 3 \mathrm{~mm}$ ), is analyzed in the context of each voxel separately. In mass-univariate analysis, a statistical inference about brain region responses to particular stimuli is made on the basis of the average activation value of a region calculated using univariate analysis conducted for each voxel in that region of a brain. Nowadays univariate and mass-univariate analysis is enhanced by Multivoxel pattern analysis (MVPA), the first concept of which appeared in the early 2000s. It also operates at a level of a voxel, however, the MVPA approach considers the fMRI analysis problem as a classification problem. Multivoxel pattern analysis has become a new paradigm for fMRI analysis in the world of neuroimaging.

The MVPA approach allows to 'decode' fMRI signals and maps them to sensory and motor events or participant's mental state [8]. A brain activity that was triggered by a certain experimental condition is recorded and represented as a pattern of voxels for that condition. In MVPA, each voxel constitutes a dimension in space, correspondingly, every pattern of voxels i.e., brain activity can be represented as a dot in that voxel space. Thus, many points (voxel patterns) form clouds in the voxel space. Figure 1 shows a simple voxel space structure: the number of dimensions was simplified to 3 voxels, red dots represent condition A and blue dots represent condition B. The plane separates two clouds. Such representation of fMRI data makes it possible to apply different supervised methods, such as support-vector machine (SVM) and linear discriminant analysis (LDA).

Functional MRI analysis is complicated by the vast number of voxels that are treated as features in classification methods. This creates a problem of "Curse of dimensionality": a number of available voxels reaches more than 30,000 , whereas the number of trials (samples) are at the highest 100 [9]. Different approaches are employed to reduce data dimensionality and thus select only 'useful' voxels. Two of the main standard approaches are region of interest (ROI) and Searchlight. Regions in ROI can be selected on the basis of anatomical structure, or on the results of application of the ANOVA method. In the latter, voxels are selected on the ANOVA test of response of each voxel to the experimental conditions [10]. After ROI selection, a functional connectivity matrix can be calculated and vectorized in different ways. Obtained low-dimensional feature vectors are then used in ML models as predictors of various diseases, see for example [11].

In the Searchlight method, a classifier is consequently trained on a small spherical cluster of voxels, centered at each voxel of the indicated areas (usually the whole brain is selected). Classification accuracies for each spherical cluster are calculated and are assigned respectively to the central voxels of the clusters. The subsets of clusters with good classification accuracy are identified, then either all voxels of these subsets are selected or the central voxels of the clusters of these subsets are selected [12]. 


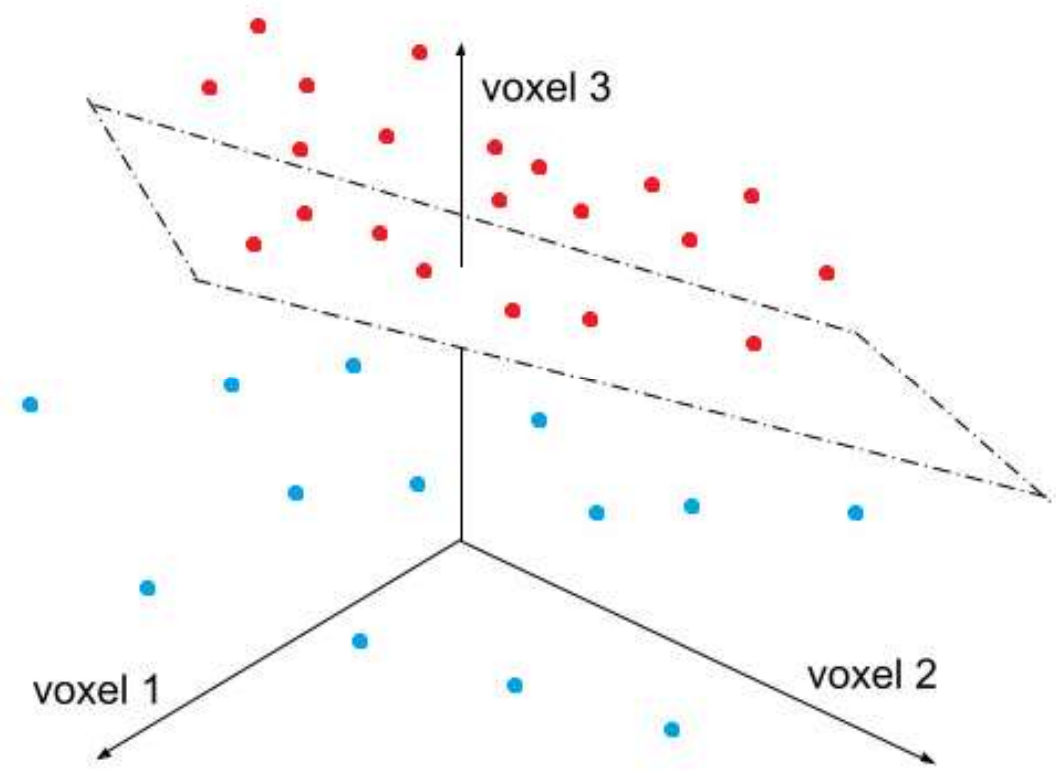

Figure 1 - Representation of brain activities of different conditions in voxel space

Common statistical approaches to dimensionality reduction, like ICA and PCA can also be successfully applied to select features. An example of ICA application is Hunyadi's [12] study of localization of epileptogenic zones, where ICA was applied to select epileptic components that later have been fed to classification algorithms [12]. Graph-theoretical approaches could be applied to fMRI-based connectivity matrices resulting in informative features for depression diagnostics [13].

Bleigh-Cohen [14] has proposed a new classification method called SBFE: patients with complex psychopathology were classified using data driven ROI search coupled with whole-brain machine-learning. SBFE showed $91 \%$ accuracy in classifying schizophrenia patients with and without OCD [14]. They state that in contrast to standard ROI driven analyses, SBFE was able to classify schizophrenia patients with and without OCD with $91 \%$ accuracy. Thus, the application of this approach can have promising results when needed to delineate patients of one complex psychiatric morbidity with the presence of different symptoms.

De Martino [15] in his work has introduced recursive feature elimination (RFE) strategy. It uses SVM reclusively to remove irrelevant voxels and assess informative spatial patterns. The method has increased sensitivity for discriminative patterns.

According to Duff [16], the number of pre-processing and feature generation methods impacts pre- diction accuracy more consistently than the choice of a classifier.

In many projects, the most popular choice of learning algorithms in fMRI analysis is SVM [17]. Among other different machine learning approaches in MVPA are Clustering algorithms [18], deep neural networks [19], and Representational Similarity Analysis [20]. In some recent work, there are first attempts to make deep neural networks models on fMRI data interpretable for particular clinical diagnostics tasks, see [21-22] for details. An advantage of using deep learning over non-deep learning approaches is that features can be automatically learned by neural networks, thus eliminating the need to conduct manual feature extraction and selection steps. Feature selection and extraction steps are performed by special structures of deep learning architecture, called convolutional and pooling layers.

In fMRI, due to the scarcity of samples compared to the number of features, the cross-validation technique is often used. Cross-validation is one of the ML techniques to improve the efficiency of the algorithm by iteratively splitting data into different training/ testing sets, and thus artificially increasing the overall training sample of the model. There are different strategies for selecting the validation fold. One of the popular strategies in MVPA is leave-subject-out or also known as leave-one-out (LOO-CV), where records of one subject are assigned to be a test dataset [23]. 
Usually, experiments in neuroscience are constructed in such a way that there is a balanced number of records with different labels in a dataset. Nevertheless, studies with unbalanced datasets can also take place: an example is the study of intrusive memory formation, in which Clark [24] attempted to predict mental health symptoms by reconstructing idiosyncratic cognitive events. The labels of the conditions were not known at the time of the experiment. Therefore, balancing techniques were needed to be employed prior to classifying the records.

As in any medical problem, sensitivity and specificity are highly important metrics for evaluating model testing results in fMRI. Accuracy itself will not be enough to provide a full picture of the effective metric. In many studies, the ROC and Precision-Recall (PR) curve is employed as an evaluation metric.

\section{Machine learning in EEG analysis}

In contrast to fMRI, EEG is an economical and easy-to-operate tool for recording brain activity [25]. It records the brain's electrical activity over a period of time. Electrochemical processes occurring in the neuronal activity of the brain resulted in electrical oscillations on the brain surface with different amplitude and frequency - alpha, beta, gamma, theta, and delta rhythms. The relationship between these rhythms depends on external stimuli and the state of the human brain. These rhythms may differ in different brain conditions. For example, Gollan's study of Frontal alpha EEG asymmetry confirms that depressed patients have a significantly higher difference in alpha rhythms between the left and right frontal part of the hemisphere than healthy participants [26].

Analysis of EEG has made it possible to develop brain-computer-interface systems. Brain-computer interface (BCI) system is an application that reads EEG signals in real-time and sends the decoded signals to an external device.

The list of the neurological disorders that can be studied using EEG signals includes but is not limited to epilepsy, seizure prediction, Alzheimer's disease, Mild Cognitive Impairment (MCI), Parkinson's disease, Creutzfeldt-Jakob Disease, sleep studies, schizophrenia, analysis of emotional states [21]. Using machine learning classification methods, it is possible to predict and prevent the development of depression [27].

EEG signals have better temporal resolution than fMRI signals, but the spatial resolution is low [28].
EEG signals are collected by electrodes placed on the participant's head. Each electrode represents an EEG channel and records a brain signal from a part of the brain that is closer to the electrode. Nevertheless, the location of an electrode may not correspond to the exact location of the brain source [29]. This constitutes an inverse problem that exists in EEG concerning localizing a brain source that elicited a particular EEG signal [30].

Although both EEG and fMRI methods employ signal processing techniques for feature extraction, it should be noted that EEG mostly employs time series processing techniques, whereas fMRI combines image processing and time-series processing techniques. In contrast to fMRI, where features are the signal intensity value at each voxel, EEG features can be EEG bands spectral powers, coherence and interhemispheric asymmetry and other possible measured parameters from time-series.

EEG analysis is conducted in either time domain or frequency domain. Time-domain techniques encompass wavelet transform and connectivity metrics, whereas frequency domain encompasses Fourier transform and further work with signal spectrum. Physical stimuli induce changes in EEG signals called Event-related potentials. These potentials can be associated with mental activity and occur during stimulus perception or preparation and execution of actions. All types of EEG parameters may serve as features for machine learning to predict the brain activity in specific conditions. For example, the authors used coherence parameters of resting state to classify depressed patients and healthy participants [31]. Machine learning is currently being applied to EEG data collected from healthy and depressed patients to predict performance differences between these two groups during a decision task [32], during an emotional regulation task [33] and vigilance objectives [34].

Deep learning approaches are becoming more popular in EEG analysis. Thus, Acharya [21] uses Convolutional Neural Network as a classifier to predict depressed or healthy people. The proposed solution by Achraya [21] used a Backpropagation algorithm to train the network, adaptive moment estimation to optimize the parameters of the network structure and dropout technique to avoid overfitting. A cross-validation strategy with 10 folds was used to test the dataset. Classification with Convolutional Neural Network has helped to achieve accuracy, sensitivity, and specificity over $90 \%$ for each left and right hemisphere. 


\section{Conclusion}

The studies reviewed in the present article demonstrate the progress that has been made due to the use of machine learning techniques in fMRI analysis, EEG analysis and in the brain signal processing field overall. With technological advances and increasing computational efficiency (Moore's law), the accuracy of the classification models in fMRI and EEG analysis may increase, even without changing the methodology of the machine learning application.

Machine learning has made enormous progress in the last two decades largely due to the growth of computing power and the emergence of deep learning; and its techniques have proved to be a valuable tool in gaining more insights from data in any domain that can possess a vast amount of data. Therefore, researchers in any field that deals with big data should be aware of the applicability and capability of machine learning techniques to be able to leverage them to their benefits. The new ML methods discovered or invented while solving cognitive science-related problems may be useful and worth considering in solving problems in other domains beyond medicine.

\section{Funding}

Research was supported by research grant from Ministry of Education and Science of Kazakhstan to A.M. Kustubayeva (AP08856595 "EEG/MRI study of brain development, emotional-cognitive functions, and genetic markers in different age groups").

M.G. Sharaev was supported by RFBR, research project 18-29-01032.

\section{References}

1. Deisenroth M.P., Faisal A.A., Ong C.S. (2021) Mathematics for Machine Learning. Cambridge University Press, 14-16 p.

2. Deo R.C. (2015) Machine Learning in Medicine. Circulation., vol. 132, no. 20, pp. 1920-1930. http://dx.doi.org/10.1161/CIRCULATIONAHA.115.001593.

3. Qayyum A., Qadir J., Bilal M., Al Fuqaha A. (2020) Secure and Robust Machine Learning for Healthcare: A Survey. IEEE Reviews in Biomedical Engineering., vol. 14, pp. 156-180. https://doi. org/10.1109/rbme.2020.3013489.

4. Nielsen A.N., Gratton C., Church J.A., Dosenbach N.U.F., Black K.J., Petersen S.E., et al.. (2019)
Atypical Functional Connectivity In Tourette Syndrome differs between Children and Adults. Biological Psychiatry., vol. 87, no. 2, pp. 164-173. http:// dx.doi.org/10.1016/j.biopsych.2019.06.021

5. Rubinov M., Sporns O. (2010). Complex network measures of brain connectivity: Uses and interpretations. NeuroImage, vol. 52, no. 3, p. 1059-1069. doi:10.1016/j.neuroimage.2009.10.003. https://doi. org/10.1016/j.neuroimage.2009.10.003.

6. Botvinick M., Wang J.X., Dabney W., Miller K.J., Kurth-Nelson Z. (2020) Deep Reinforcement Learning and Its Neuroscientific Implications. Neuron, vol. 107, no. 4, pp. 603-616. https://doi. org/10.1016/j.neuron.2020.06.014.

7. Niv Y. (2009). Reinforcement learning in the brain. Journal of Mathematical Psychology., vol. 53, no. 3, pp. 139-154.https://doi.org/10.1016/j. jmp.2008.12.005.

8. Haynes J-D, Rees G. (2006) Decoding mental states from brain activity in humans. Nature Reviews Neuroscience., vol. 7, no. 7, pp. 523-534. http:// dx.doi.org/10.1038/nrn1931.

9. Hirose S, Nambu I, Naito E. (2015) An empirical solution for over-pruning with a novel ensemblelearning method for fMRI decoding. Journal of Neuroscience Methods., vol. 239, pp. 238-245. http:// dx.doi.org/10.1016/j.jneumeth.2014.10.023.

10. Jamshy S., Perez O., Yeshurun Y., Hendler T., Intrator N. (2012) Searchlight Based Feature Extraction. In: Langs G., Rish I., Grosse-Wentrup M., Murphy B. (eds) Machine Learning and Interpretation in Neuroimaging. Lecture Notes in Computer Science, vol. 7263. Springer, Berlin, Heidelberg. https://doi. org/10.1007/978-3-642-34713-9_3.

11. Sharaev M., Artemov A., Kondrateva E., Sushchinskaya S., Burnaev E., Bernstein A., et al. (2018) MRI-Based Diagnostics of Depression Concomitant with Epilepsy: In Search of the Potential Biomarkers. Proceedings of IEEE international conference on data science and advanced analytics. Turin, Italy, P. 555. https://www.doi.org/10.1109/DSAA.2018.00071.

12. Hunyadi B, Tousseyn S, Dupont P, Van Huffel S, De Vos M, Van Paesschen W. (2015) A prospective fMRI-based technique for localising the epileptogenic zone in presurgical evaluation of epilepsy. NeuroImage., vol. 113, pp. 329-339. http://dx.doi. org/10.1016/j.neuroimage.2015.03.011.

13. Sharaev M., Artemov A., Kondrateva E., Ivanov S., et al. (2018) Learning Connectivity Patterns via Graph Kernels for fMRI-Based Depression Diagnostics. Proceedings of IEEE international conference on data mining workshops. Singapore, Sin- 
gapore, P. 308. https://www.doi.org/10.1109/ICDMW.2018.00051.

14. Bleich-Cohen M., Jamshy S., Sharon H., Weizman R., Intrator N., Poyurovsky, M., et al. (2014) Machine learning fMRI classifier delineates subgroups of schizophrenia patients. Schizophrenia Research., vol. 160 no. 1-3, pp. 196-200. http:// dx.doi.org/10.1016/j.schres.2014.10.033.

15. De Martino F, Valente G, Staeren N, Ashburner J, Goebel R, Formisano E. (2008) Combining multivariate voxel selection and support vector machines for mapping and classification of fMRI spatial patterns. NeuroImage., vol. 43, no. 1, pp. 44-58. http://dx.doi.org/10.1016/j.neuroimage.2008.06.037.

16. Duff K. (2012) Evidence-Based Indicators of Neuropsychological Change in the Individual Patient: Relevant Concepts and Methods. Archives of Clinical Neuropsychology., vol. 27, no. 3, pp. 248261. http://dx.doi.org/10.1093/arclin/acr120.

17. Yamamoto M, Bagarinao E, Kushima I, Takahashi T, Sasabayashi D, Inada T, Suzuki M, Iidaka T, Ozaki N. (2020) Support vector machine-based classification of schizophrenia patients and healthy controls using structural magnetic resonance imaging from two independent sites. PLoS One., vol.15, no. 11. https://doi.org/10.1371/journal.pone.0239615.

18. Aljobouri H.K., Jaber H.A., Koçak O.M., Algin O, Çankaya I. (2018) Clustering fMRI data with a robust unsupervised learning algorithm for neuroscience data mining. Journal of Neuroscience Methods., vol. 299, pp. 45-54. http://dx.doi.org/10.1016/j.jneumeth.2018.02.007.

19. Svanera M, Savardi M, Benini S, Signoroni A, Raz G, Hendler T, et al. (2019) Transfer learning of deep neural network representations for fMRI decoding. Journal of Neuroscience Methods., vol. 328, 108319. https://doi.org/10.1016/j.jneumeth.2019.108319.

20. Kriegeskorte N. (2008) Representational similarity analysis - connecting the branches of systems neuroscience. Frontiers in Systems Neuroscience., vol. 2, no 4., http://dx.doi.org/10.3389/neuro.06.004.2008.

21. Li X., Dvornek N.C., Zhou Y., Zhuang J., Ventola P., Duncan J.S. (2019) Graph Neural Network for Interpreting Task-fMRI Biomarkers. Proceedings of international conference on medical image computing and computer assisted intervention. Shenzhen, China, P. 485.

22. Yan W., Calhoun V., Song M., Cui Y., et al. (2019) Discriminating schizophrenia using recurrent neural network applied on time courses of multi-site
FMRI data. EBioMedicine. https://doi.org/10.1016/j. ebiom.2019.08.023.

23. Lueken U, Straube B, Yang Y, Hahn T, Beesdo-Baum K, Wittchen H-U, et al. (2015) Separating depressive comorbidity from panic disorder: A combined functional magnetic resonance imaging and machine learning approach. Journal of Affective Disorders., vol. 184, pp. 182-192. http://dx.doi. org/10.1016/j.jad.2015.05.052.

24. Clark I.A., Niehaus K.E., Duff E.P., Di Simplicio M.C., Clifford G.D., Smith S.M., et al. (2014) First steps in using machine learning on fMRI data to predict intrusive memories of traumatic film footage. Behaviour Research and Therapy., vol. 62, pp. 37-46. http://dx.doi.org/10.1016/j.brat.2014.07.010.

25. Acharya U.R., Oh S.L., Hagiwara Y., Tan J.H., Adeli H., Subha D.P. (2018) Automated EEGbased screening of depression using deep convolutional neural network. Computer Methods and Programs in Biomedicine., vol. 161, pp. 103-113. http:// dx.doi.org/10.1016/j.cmpb.2018.04.012.

26. Gollan J.K., Hoxha D., Chihade D., Pflieger M.E., Rosebrock, L., Cacioppo, J. (2014). Frontal alpha EEG asymmetry before and after behavioral activation treatment for depression. Biological Psychology., vol. 99, pp. 198-208. https://doi.org/10.1016/j. chiabu.2020.104358.

27. Bachmann M, Päeske L, Kalev K, Aarma K, Lehtmets A, Ööpik P, et al. (2018) Methods for classifying depression in single channel EEG using linear and nonlinear signal analysis. Computer Methods and Programs in Biomedicine., vol. 155, pp. 11-17. http://dx.doi.org/10.1016/j.cmpb.2017.11.023.

28. Liley D.T.J., Frascoli F. (2010) Approaches to Modelling the Dynamical Activity of Brain Function Based on the Electroencephalogram. In: Dewar R.L., Detering F. (eds) Complex Physical, Biophysical and Econophysical Systems. World Scientific Lecture Notes in Complex Systems, vol. 9. World Scientific Publishing Co., Singapore, Singapore. https://doi. org/10.1142/9789814277327 000.

29. Teplan M. (2002) Fundamentals of EEG measurement. Measurement Science Review., vol. 2, no. 2, pp. 1-11

30. Michel C.M., Brunet D. (2019) EEG Source Imaging: A Practical Review of the Analysis Steps. Frontiers in Neurology., vol 10. https://doi. org/10.3389/fneur.2019.00325

31. Ledovsky A., Mnatsakanian E., Sharaev M., Burnaev E. (2019) O-21 Separation of major depression patients from healthy controls using machine learning approach to resting-state EEG. Clinical 
Neurophysiology., vol. 130, no. 7, p. e28. https://doi. org/10.1016/j.clinph.2019.04.337.

30. Kustubayeva A., Kamzanova A., Kudaibergenova S., Pivkina V., Matthews G. (2020) Major depression and brain asymmetry in a decision-making task with negative and positive feedback. Symmetry., vol. 12, no. 12, pp. 1-25. https://doi.org/10.3390/ sym12122118.

33. Kustubayeva A., Tolegenova A., Matthews G. (2013) EEG-brain activity in different strategies of emotions' self-regulation: Suppression and reappraisal. Psikhologicheskii Zhurnal.,, 34(4), pp. 58-68. https://doi.org/10.3390/brainsci9090225.

34. Kamzanova A., Kustubayeva A., Matthews G. (2012) Diagnostic monitoring of vigilance decrement using EEG workload indices. Proceedings of the Human Factors and Ergonomics Society Annual Meeting. Boston, Massachusetts, USA. P. 203. https://doi.org/10.1177/1071181312561019. 